\title{
A Hierarchical Depth Buffer for Minimizing Memory Bandwidth in 3D Rendering Engine: DEPTH FILTER
}

\author{
Chang-Hyo $Y u$ and Lee-Sup Kim \\ Department of EECS, KAIST, 373-1 Guseong-dong, Yuseong-gu, Daejeon, 305-701, Republic of Korea \\ E-mail:\{mosmov,lskim\}@mvlsi.kaist.ac.kr
}

\begin{abstract}
In this paper, we propose a new class of hierarchical depth test which saves memory bandwidth in 3D graphics rendering engine by reducing the number of pixels being passed to the per-pixel operation pipeline. This new filtering, Depth Filter, can be implemented by adding a simple hardware in front of the perpixel operation pipeline. The Depth Filter is a filtering block which decides whether a pixel is shaded by certain plane. The plane is the mask which has the history that a pixel has appeared in front of the plane. If the pixel is shaded, the pixel can be removed. The simulation shows that Depth Filter reduces the number of pixels to the next stage up to 62.1 percent in random scene. As a result, $62.1 \%$ of memory bandwidth is saved with simple extra hardware.
\end{abstract}

\section{INTRODUCTION}

Consumer-oriented 3D graphics hardware rapidly incorporates algorithms that increase flexibility and quality of per-pixel color processing; a recent example is a pixel shader [1]. However, determination if a pixel is visible and needs to be processed by a shader still relies on the screen $Z$ buffer algorithm [2]. If the scene is more and more complex, depth buffer test also becomes a burden to the rendering engine. Consider, for example, an interactive walk-through of a detailed geometric database describing an entire city, complete with vegetation, buildings, furniture inside the buildings and the contents of the furniture. Traditional visibility test which runs on curent graphics hardware, the screen $\mathrm{Z}$ buffer algorithm, is not capable of rendering very complex images at interactive rates because of the shortage of memory bandwidth. The vast memory bandwidth is used in the $\mathrm{Z}$ buffer algorithm because every pixel requests the depth value stored in the depth buffer and the depth of every pixels survived in the depth test must be updated to the depth buffer.

There are Texture Read(TR), Depth Buffer Read(RZ), Depth Buffer Write(WZ), Color Read(RC), Color Write(WC) of memory requests in the $3 \mathrm{D}$ graphics rendering engine. $\mathrm{RZ}, \mathrm{WZ}$, $W C$ and $T R$ are usually requested to access memory in order to render one pixel. In the worst case, all of these memory accesses are necessary. Only $R Z$ test are required in the case that a pixel is rejected in depth test, that is to say, the best case. Since every request, TR, RZ, WZ, RC and WC is usually 4 bytes, the usual case requests about 16 bytes/second of memory bandwidth. For example, NVIDIA's GeForce2 Uitra [3] generates 1.0Gigapixels every second in the peak performance. However, the performance is usually limited by given memory bandwidth, $7.36 \mathrm{~GB} / \mathrm{sec}$, because $16 \mathrm{~GB} / \mathrm{sec}((\mathrm{RZ}+\mathrm{WZ}+\mathrm{WC}+\mathrm{TR}) \times 1 \mathrm{G}=16 \mathrm{~GB} / \mathrm{sec})$ memory bandwidth is required to render pixels with the peak performance in ordinary case (RZ, WZ, WC and TR are needed).
As shown in the preceding paragraph, the memory bandwidth causes one of the most critical bottlenecks in 3D graphics hardware. Therefore, there has been lots of research done to resolve this memory bandwidth problem. These research approaches are separated into two ways. The first way is to reduce the number of objects in the geometry part [4]. Though this approach acquires much saving of memory bandwidth, it requires a pre-processing work to build the database of geometric data. Because the pre-processing work is too expensive to implement in hardware, there has been no hardware which implements this method. The second way is an approach to reduce the bandwidth in the rendering part. Although this method is not better than the first in performance, it is simple to implement. For example, texture compression [5] and texture cache [6] are generally used in current hardware. Hyper-Z [7], ATI's commercial hardware, reduces the memory requests by using compression, block management and hierarchical approaches but Hyper- $Z$ is not open to the public in this time

We present a very simple solution to perform the depth test partially before other memory accesses are requested. In the conventional method, even the invisible pixels, which are doomed to be hidden by another pixel which is closer to an eyepoint, are passed through the per-pixel pipelines and rejected by the depth test in the end. The operations which are needed for these invisible pixels waste the memory bandwidth. Therefore we reject the invisibie pixels, which can be decided by our algorithm. earlier as many as possible. In the simulation, the Depth Filter reduces up to $62.1 \%$ of total pixels.

In the next section, we show the basic idea. Section 3 describes the simulation environment and results. Section 4 gives analysis on the results. Finally, in Section 5, we conclude this paper.

\section{DEPTH FILTER}

\subsection{Depth buffer}

Recent 3D graphics hardware generally uses a hardwired depth buffer for visibility test. There are some types of depth buffers [8]. Among these buffers, Z-buffer is the most popular method. It uses transformed $z$-coordinate which is a transformed value from eye-coordinate to screen-cootdinate. It determines which pixel is visible by the comparison of $z$ value. In other words, it stores a closest pixel from the eye-point and the others are removed. It can be implemented very easily. 


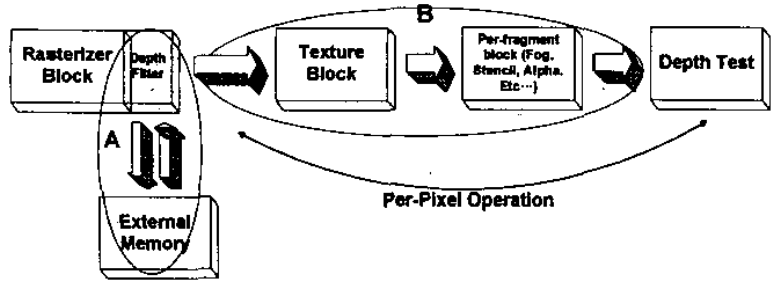

Figure 1 : Rendering Engine Block Diagram

Figure 1 shows a block diagram of the simplified rendering engine of 3D graphics pipeline. Block $A$ is the newly added unit for the depth filter and the other blocks are the conventional graphics pipeline. Block $B$ is the per-pixel operation blocks which can be reduced the total operations by the depth filter for unnecessary pixels.

\subsection{Depth Filter}

\subsubsection{Depth Plane}

In recent 3D graphics pipeline, the depth test block is located in the rear of the per-fragment block. If we move the depth test to the front block, for example the rasterizer, we can save the memory bandwidth because this method prevents the per-pixel pipeline from wasting of memory accesses for the unnecessary pixels. But in this case, we are confronted by huge data traffic [9], which is composed of texture operations, alpha, stencil test and etc. between the rasterizer and the conventional depth test block as shown in the figure.1. Because of this expensive overhead, we cannot move the depth test to the rasterizer. Therefore we use the hierarchical concept that the Depth Filter performs a pre-depth test in the rasterizer. This newly added block uses only 1 bit or 2 bits comparison instead of real 24 bits or 32 bits precision like a conventional depth buffer. Therefore it does not cause the overhead as stated above. By this simple test, we have large portions of pixels rejected. It is the main advantage of our method.

Our method needs the information that a pixel is located in front or back of the plane (one-plane system). This information is used for setting the Depth Filter's data and for testing whether the pixel is hidden by the plane or not. If the pixel fails the Depth Filter test, pass to the conventional pipelines and then the depth buffer block tests this pixel one more time by 24 bits or 32 bits.

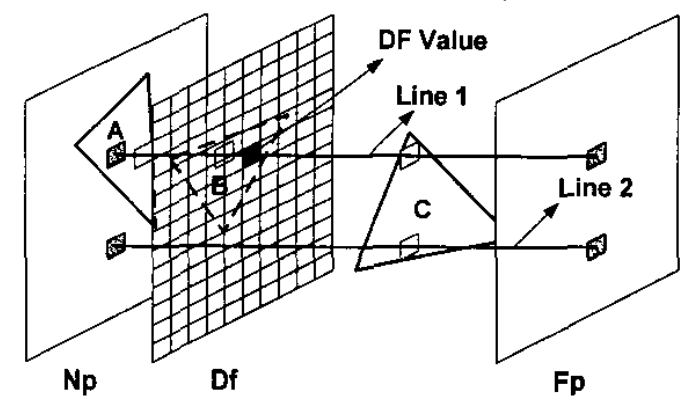

Figure 2 : Depth Filter (One-Plane). Points with white-rectangle are on the triangle. Points with gray-rectangle are on the plane.
The position of the plane is set by the $z$ coordinate in screencoordinates. If a $z$ value is smaller than DF (the position of the depth plane), the value of that pixel, in Depth Filter, is set " 1 ". It means the object is closer to the eye or NP (Near Plane) than DF. If a $\mathrm{z}$ value is lager than DF, the Depth Filter investigates the history of current coordinate. And then if the value is " 1 ", current pixel is rejected. And in the other case, pixels are passed to the conventional graphics pipelines (per-pixel operation).

Assume the triangle $\mathrm{A}, \mathrm{B}$ and $\mathrm{C}$ are rendered in consecutive order in figure 2. In line 1 , the triangle $A$ precedes DF. Therefore the flag of current coordinate is set " 1 " shown by (DF value) a rectangle point in the DF. The triangle $B$ is rendered between the triangle $A$ and DF. Because the triangle $B$ is also in front of DF, the Depth Filter is updated like that of the triangle A. And at last, the triangle $C$ is rendered behind DF. In this case, because the value of Depth filter for that pixel is " 1 " which is set before by the pixel on the same line in the triangle $A$ and $B$, the Depth Filter decides that the pixel is hidden by DF. Then this pixel is rejected. In line2, the pixel on the triangle $C$ is only on the line. This pixel is rendered with the examination of Depth Filter. But the value of that pixel in DF is " 0 " because that pixel is never been updated. Therefore the pixel must be passed to the conventional rendering pipeline. As the pixel on the line 2 , in the triangle $\mathrm{C}$, is behind DF, Depth Filter is not updated.

To make simple hardware, Depth Filter is implemented in two layers. In the first layer, SRAM is used for internal storing unit. It behaves like a cache memory in CPU. And the second layer is composed of DRAM on the outside. The external memory has the entire screen data of the Depth Filter. The internal SRAM uses these screen data like a cache with the line size of 32 or 64 pixel-block and 8 slots.

Because the access to external memory is very expensive, we must try to reduce this access. Therefore we try to increase the ratio of pixels rejected by Depth Filter and the ratio of internal SRAM hit to reduce the access. The former is acquired by adding the number of planes. The latter is acquired by increasing the internal SRAM size. Because the rejection ratio is more important, the number of planes is more effective. We add 1 bit for each pixel to increase a plane. Therefore Depth Filter has 2 bits storage, which can store the four values, for each pixel. These four values indicate each space between the planes (11: DF 1, $10:$ DF2, $01:$ DF3, 00 : none ).

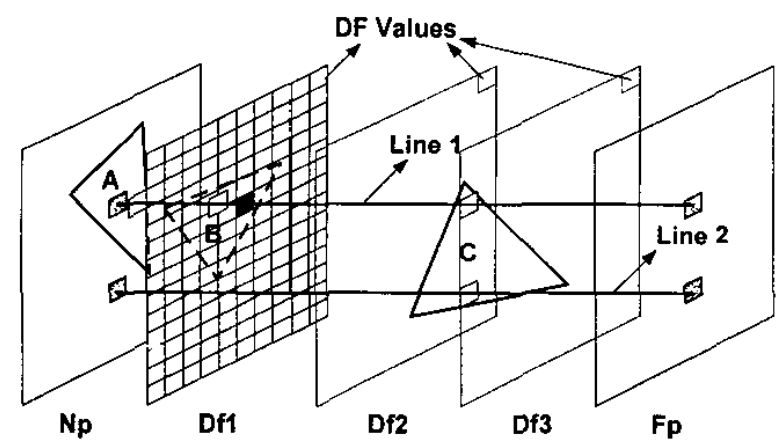

Figure 3 : Depth Filter (Three-Plane) 
Figure 3 shows the three-plane Depth Filter system. The point that is on the Triangle C can be rejected if the DF2's value of that pixel is " 1 " unlike the one-plane system in the figure 2 .

\subsubsection{Plane Decision}

Plane decision is very important in Depth Filter because the position of plane decides the number of pixels which can be tested. Most of the objects in typical 3D scenes are located close to near plane. And $\mathrm{Zdf}$, as in the figure 4 , is also converged to near plane. Therefore we can use the most of the range $[0,1]$.

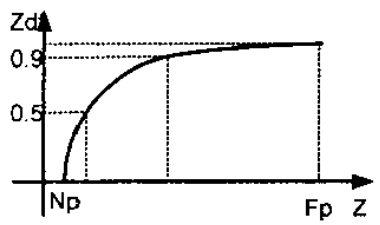

Figure 4 : Z-Coordinates (Zdf)

In this time, we test all the cases with DF from 0.1 to 0.8 . And we get the statistics from random cases. We choose $\mathrm{DF}=0.35$ in one-plane system from the statistics.

In the three-plane system, Depth Filter uses the position of planes which are DF $1=0.15, \mathrm{DF} 2=0.35$ and DF $3=0.55$. The position of DF2 is the same as the position of plane in the one-plane system because the distributions of objects are the same. And DF1 and DF2 increase the filtering coverage and enable the comparison in detail. In the three-plane system, the positions of plane are less important than the one-plane system because it covers the most part of the object space. Therefore it is better to use the three-plane system

\section{SIMULATION \& RESULT}

\subsection{Simulation}

There is a software library, Mesa [10], which is widely used for 3D graphics. But it does not support a hardware-like modeling and simulation environment. To test our algorithm with easy and low level block description, we use the GATE [11] (Graphics Architecture Testing Environment) which is the testing environment designed for easy modification and verification of 3D graphics pipeline based on Microsoft Visual $\mathrm{C}+$.

\section{Internal SRAM Spec}

- Fully Associative method

- Line Sizc $=>32$ or 64-bit block

- SRAM slot $=>8$ slots $($ Line $\times 8)$

- Tag $\Rightarrow>6+6$-bit (12 bytes)

- Counter + Valid $=>4$-bit $(4$ bytes $)$

- LFU replacement policy

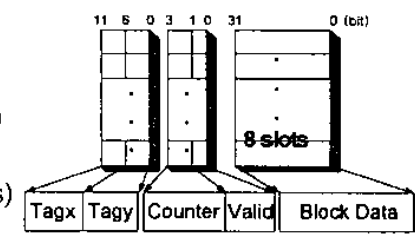

Figure 5 : Internal SRAM Block Diagram

External DRAM Spec

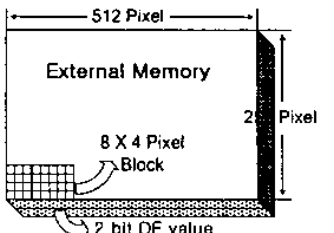

Figure 6 : External Memory Block Diagram.
Figure 6 shows the external memory. If we want to use 64-bit block, we can change the $8 \times 4$ pixel block to $8 \times 8$ pixel block.

A pseudo code for Depth Filter is as follows.

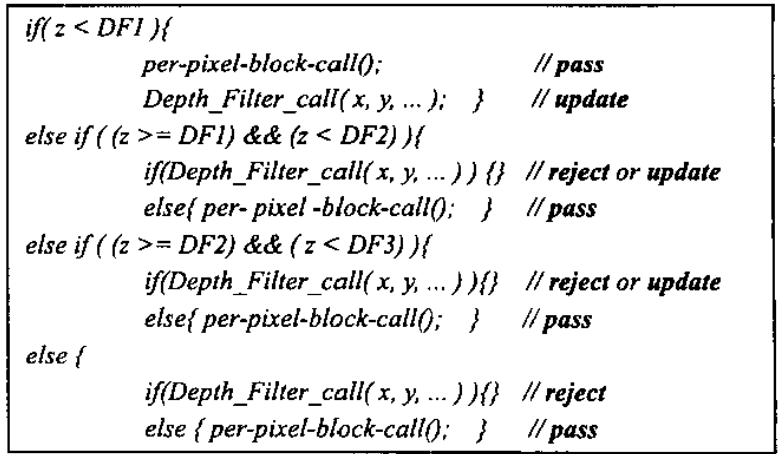

\subsection{Result}

These results are from the two test scenes in figure 7. the figure 7 (a) is composed of 200 hexagonal columns generated randomly and the figure 7(b) is a normal 3D model of tree. These results are not the best cases. They are only random case results.
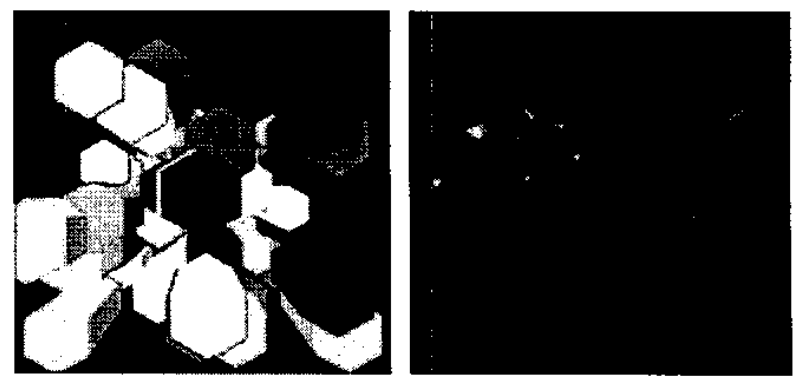

(a) Random object(799963 pixels) (b) A tree(581177 pixels) Figure 7 : Test Scenes.

\begin{tabular}{|l|c|c|c|}
\hline $\begin{array}{c}\text { Plane / } \\
\text { block size(bit) }\end{array}$ & $\begin{array}{c}\text { Rejection } \\
\text { Ratio }\end{array}$ & $\begin{array}{c}\text { SRAM Hit } \\
\text { Ratio }\end{array}$ & $\begin{array}{c}\text { Effective } \\
\text { Depth Test } \\
\text { Ratio }\end{array}$ \\
\hline \multicolumn{4}{|c|}{ Scene (a) } \\
\hline 1-plane / 32 & 0.421072 & 0.938259 & 0.590879 \\
\hline 1-plane / 64 & 0.466906 & 0.965205 & 0.649076 \\
\hline 3-plane / 32 & 0.535926 & 0.938016 & 0.647649 \\
\hline 3-plane / 64 & 0.621597 & $\mathbf{0 . 9 6 5 1 6 2}$ & $\mathbf{0 . 7 0 9 3 7 5}$ \\
\hline \multicolumn{4}{|c|}{ Scene (b) } \\
\hline 1-plane / 32 & 0.336036 & 0.923297 & 0.608180 \\
\hline 1-plane / 64 & 0.336198 & 0.959155 & 0.608220 \\
\hline 3-plane / 32 & 0.436131 & 0.923614 & 0.668158 \\
\hline 3-plane / 64 & 0.437205 & $\mathbf{0 . 9 5 9 3 6 2}$ & 0.668703 \\
\hline
\end{tabular}

Table 1 : Results from test scenes.

Rejection Ratio: The ratio of the rejected pixels by Depth Filter. The portions of (1-Rejection Ratio) are passed to conventional pipeline.

SRAM Hit Ratio: The ratio of that Internal SRAM has the needed pixels of Depth Filter. The portions of (1- Hit Ratio) are needed external memory accesses. 
Effective Depth Test Ratio: The ratio of rejected pixels from Depth Filter to rejected pixels from conventional depth test. It works like a pre-depth buffer test.
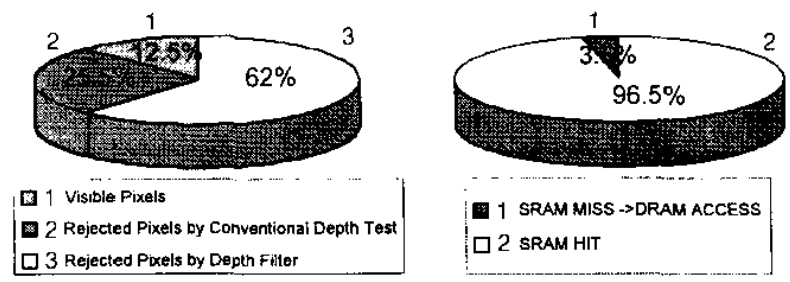

Figure 8 : Result from 3-plane/64 block in Test Scene (a)

In figure 8 , the left graph shows that $62 \%$ of total pixels are rejected by the Depth Filter and $38 \%$ of total pixels are passed to the per-pixel pipeline. Among the passed pixels, only $12.5 \%$ are displayed and the others are rejected by the conventional depth test. The right graph shows that $96.5 \%$ of Depth Filter's values are in the internal SRAM and the others need an external memory access.

\section{DISCUSSION}

More than $60 \%$ of the total pixels are rejected by Depth Filter in the best case. Therefore the required memory bandwidth is reduced to $40 \%$ of the traditional hardware. Although the memory bandwidth is required for SRAM miss which requests a external memory access, the external memory access needs only 4 bytes for one block and needs only about $3 \sim 6 \%$ (SRAM miss ratio) of total calls of the Depth Filter. Therefore the required bandwidth for the Depth Filter is $55.2 \sim 110.4 \mathrm{Mbytes} / \mathrm{sec}$. (230 $\mathrm{MHz} \times 2 \times 4$ bytes $\times(0.03 \sim 0.06)=55.2 \sim 110.4 \mathrm{MB} / \mathrm{s})$. In this case, the bandwidth gain, the effective memory bandwidth over the traditional memory bandwidth, is 2.485 . (the effective memory bandwidth is $7.36 \mathrm{~GB} / \mathrm{s}$ [3] $\times 100 \% /(100 \%-60 \%)-$ $110.4 \mathrm{MB} / \mathrm{s}=18.29 \mathrm{~GB} / \mathrm{s})$

These results are from the various simulations with different number of planes and different size of SRAM. Because the planes affect the number of pixels to be tested, the number of planes affects the total rejection ratio. The size of SRAM affects SRAM hit ratio because of the storage capacity in SRAM. These variables are highly related to cost-effectiveness.

The position of the filter is important to get a good result. In this paper, we used the position from the statistics which are from the random objects. If we change the position with adaptively, Depth Filter could get a good result independent with the distribution of object but this work is remained to a future work.

To implement in hardware, we must consider the external memory specifications. Nowadays, for example, commercial graphics hardware uses 128-bit DDR SDRAM. In this case, the size of block is to be 128 bits because a basic memory transfer size of DDR SDRAM is 128 bits. (In our simulations, Depth Filter used 32-bit or 64-bit data block) Since the external memory does not have the limit of the size compared to SRAM in recent technique, we can enlarge the screen size by increasing the external memory and use various managing schemes
Even when the scene has a low depth complexity (Test Scene (b)), Depth Filter still acquires large portions of pixels rejected (over $40 \%$ at the 3-plane system) and has good result of effective depth test ratio. It means that it is also effective to reduce the number of depth tests in the conventional depth test. Although Test Scene (b) cannot get as good results of the rejection ratio as Test Scene (a), it still reduces the number of depth tests exceeding $60 \%$.

\section{CONCLUSIONS}

As more complex models become commonplace in 3D computer graphics, it. becomes increasingly important to remove the unnecessary operations which waste the memory bandwidth. In this paper, we present an algorithm which saves memory bandwidth in Rendering Engine of $3 \mathrm{D}$ graphics hardware.

Depth Filter is a filtering method which removes the invisible pixels ahead of per-pixel pipelines. It reduces the per-pixel operations to cause lack of the memory bandwidth by adding an extra hardware into the rasterizer that is placed in front of the per-pixel pipeline. Up to $62.1 \%$ of total pixels were removed by this method for the complex test scenes. In other words, the bandwidth gain obtained by Depth Filter is 2.624 .

Depth Filter can be easily implemented in the conventional 3D graphics hardware such that it can be a useful method to solve the memory bottlenccks in the 3D Rendering Engine.

\section{REFERENCES}

[1] Sim Dietrich. DX8 Pixel Shaders. Presentation at the GDC'2001, San Jose, March 2001 http:/www.nvidia.com/Marketing/Developer/DevRel.nsf/bo okmark/8A8AB1C90C2B8A4388256A1800662B3B

[2] W.M. Newmann, R.F. Sproull Principles Of Interactive Computer Graphics. 1981 New York: McGraw-Hill

[3] NVIDIA Corporation, http://www.nvidia.com/docs/lo/1048/ SUPP/gf2_overview.pdf

[4] H. Zhang, D. Manocha, T. Hudson, K.E. Hoff III, Visibility Culling using Hierarchical Occlusion Maps. SIGGRAPH, 1997, pp.77-88

[5] S3TC DirectX 6.0 Standard Texture Compression, Savage 3D white papers, 1998

[6] Hakura, Z.S., Gupta, A, The Design and Analysis of a Cache Architecture for Texture Mapping. The 24th Annual International Symposium on Computer Architecture, 1997, pp. $108-120$

[7] Steve Morein, ATI Radeon HyperZ Technology, 2001, ATI Corporation, http://www.ati.com

[8] Lapidous E, Jiao G. Optimal Depth Buffer For Low-Cost Graphics Hardware. SIGGRAPH/Eurographics Workshop on Graphics Hardware, 1999, pp. 67-73.

[9] J. McCormack, R. McNamara, C. Gianos, L. Seiler, N. Jouppi, K. Correll, Neon: a single-chip 3D workstation graphics accelerator, SIGGRAPH/Eurographics Workshop on Graphics Hardware, August 1998, pp 123-132.

[10] Mesa, http://www.mesa3d.org

[11] GATE , http://mvlsi.kaist.ac.kr/project/gate/gate.htm 